\title{
The 1998 outburst of the X-ray transient XTE J2012+381 as observed with BeppoSAX
}

\author{
S. Campana ${ }^{1}$, L. Stella ${ }^{2, \star}$, T. Belloni ${ }^{1}$, G. L. Israel ${ }^{2, \star}$, A. Santangelo ${ }^{3}$, F. Frontera ${ }^{4,5}$, \\ M. Orlandini ${ }^{5}$, and D. Dal Fiume ${ }^{5}$ \\ 1 Osservatorio Astronomico di Brera, via E. Bianchi 46, 23807 Merate (Lecco), Italy \\ 2 Osservatorio Astronomico di Roma, via Frascati 33, 00040 Monteporzio Catone (Roma), Italy \\ 3 Istituto di Fisica Cosmica e Applicazioni all'Informatica, C.N.R., via La Malfa 153, 90146 Palermo, Italy \\ 4 Università di Ferrara, Dipartimento di fisica, via Paradiso 12, 44100 Ferrara, Italy \\ ${ }^{5}$ Istituto Tecnologie e Studio Radiazioni Extraterrestri, C.N.R., via Gobetti 101, 40129 Bologna, Italy \\ Received 8 November 2001 / Accepted 14 December 2001
}

\begin{abstract}
We report on the results of a series of X-ray observations of the transient black hole candidate XTE J2012+381 during the 1998 outburst performed with the BeppoSAX satellite. The observed broad-band energy spectrum can be described by the superposition of an absorbed disk black body, with an iron line plus a high energy component, modelled with either a power law or a Comptonisation tail. The source showed pronounced spectral variability between our five observations. While the soft component in the spectrum remained almost unchanged throughout our campaign, we detected a hard spectral tail which extended to $200 \mathrm{keV}$ in the first two observations, but became barely detectable up to $50 \mathrm{keV}$ in the following two. A further re-hardening is observed in the final observation. The transition from a hard to a soft and then back to a hard state occurred around an unabsorbed $0.1-200 \mathrm{keV}$ luminosity of $10^{38} \mathrm{erg} \mathrm{s}^{-1}$ (at $10 \mathrm{kpc}$ ). This indicates that state transitions in XTE $2012+281$ are probably not driven only by mass accretion rate, but additional physical parameters must play a role in the evolution of the outburst.
\end{abstract}

Key words. binaries: general - black hole physics - stars: individual (XTE J2012+381) - X-rays: stars

\section{Introduction}

The transient X-ray source XTE J2012+381 was discovered with the Rossi X-ray Timing Explorer All Sky Monitor (RXTE-ASM) on May 24, 1998 at a level of $23 \mathrm{mCrab}(2-12 \mathrm{keV}$; Remillard et al. 1998; see Fig. 1). Within 3 days the source raised to an average level of $88 \mathrm{mCrab}$ (May 27, 1998), reaching values as high as $110 \mathrm{mCrab}(2-10 \mathrm{keV}$, RXTE Proportional Counter Array - PCA; Marshall \& Strohmayer 1998). An ASCA observation on May 29, 1998 (MJD 50962) showed the source at a level $150 \mathrm{mCrab}(2-10 \mathrm{keV}$; White et al. 1998). The ASCA Gas Imaging Spectrometer $0.5-10 \mathrm{keV}$ spectrum could be well described by the superposition of a multicolor disk black body and a power law model. The temperature of the innermost disk radius was $k T=0.76 \pm 0.01 \mathrm{keV}$, the power law photon index $\Gamma=2.9 \pm 0.1$ and the absorption column density $N_{\mathrm{H}}=(1.29 \pm 0.03) \times 10^{22} \mathrm{~cm}^{-2}$ (White et al. 1998). The presence of a soft thermal component (equivalent temperature $\sim 1 \mathrm{keV}$ ) plus a hard power law is considered to bea characteristic feature of

Send offprint requests to: S. Campana,

e-mail: campana@merate.mi.astro.it

* Affiliated to I.C.R.A. black hole candidates (e.g. Tanaka \& Shibazaki 1996). The outburst evolution as observed by the RXTE-ASM is shown in Fig. 1. An extensive analysis of the available RXTE PCA pointings was carried out by Vasiliev et al. (2000).

Very Large Array (VLA) observations on 31 May, 1998 led to the identification of a radio source (Hjellming et al. 1998a). The radio source was located at $\mathrm{RA}=$ $20^{\mathrm{h}} 12^{\mathrm{m}} 37^{\mathrm{s}} .67$ and Dec $=+38^{\circ} 11^{\prime} 01^{\prime} \cdot 2$ (equinox 2000) within the $\sim 1^{\prime}$ RXTE $90 \%$ error circle (Hjellming et al. 1998b).

Despite the large column density (converting to a $V$-band extinction of about $7 \mathrm{mag}$ ) inferred from the $\mathrm{X}$-ray spectrum, the large flux variations characteristic of X-ray novae made possible the identification of a faint red $(R=20.1 ; V=21.3)$ counterpart during the X-ray source outburst, at a position consistent with the location of the radio counterpart (Hynes \& Roche 1998; Hynes et al. 1999).

Here we report on an observation campaign carried out with the Italian-Dutch satellite BeppoSAX (Boella et al. 1997a), aimed at studying in detail the outburst of XTE J2012+381. Five observations were carried out starting from May 28, 1998 through July 8, 1998. The $\mathrm{X}$-ray spectra and light curves are discussed in Sect. 2. 


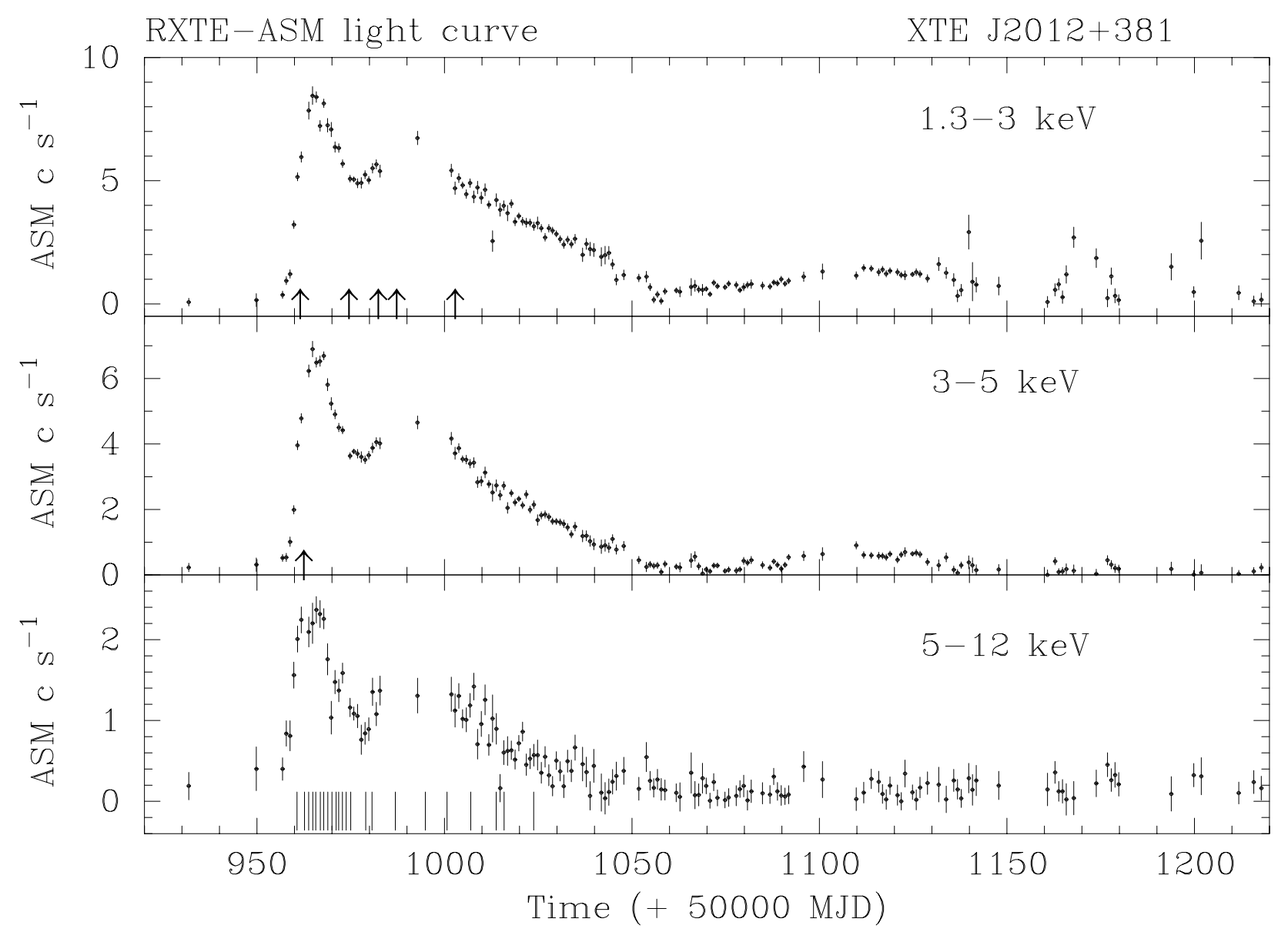

Fig. 1. X-ray light curve of XTE J2012+381 as observed with the RXTE-ASM in the three energy band (1.3-3, 3-5 and 5-12 keV). The arrows in the upper panel mark the epochs of the five BeppoSAX observations; the arrow in the middle panel marks the epoch of the ASCA observation whereas the bars at the bottom of the lower panel show the epochs of the RXTE pointed observations.

Section 3 is dedicated to the discussion of the results; our conclusions are summarised in Sect. 4 .

\section{Data analysis}

We analysed the data from all the BeppoSAX narrow field instruments: the Low Energy Concentrator Spectrometer (LECS; 0.1-10 keV, Parmar et al. 1997), the Medium Energy Concentrator Spectrometer (MECS; 1.3-10 keV, Boella et al. 1997b), the High Pressure Gas Scintillation Proportional Counter (HPGSPC; 4-120 keV, Manzo et al. 1997) and the Phoswich Detection System (PDS; 15-300 keV, Frontera et al. 1997). At the time of the observations, only two of the three MECS units were operating. LECS data were collected only during satellite night-time leading to substantially shorter exposure times compared to the MECS. The HPGSPC and PDS collimators were alternatively rocked on and off the source to monitor the background, effectively halving their exposure times. During an additional BeppoSAX observation that took place on MJD 50967 the MECS detectors were turned off and the satellite experienced several drifts resulting in different source locations in the focal plane. We excluded this observation from further analysis.

\subsection{Spectral analysis}

The LECS and MECS data were extracted within a radius of $8^{\prime}$ centered on the source position. Background subtraction was applied even if its influence is only marginal (it comprises $99.9 \%$ of the total flux). We rebinned the LECS and MECS spectra using the grouping files provided by SAX-SDC, lecs_5.grouping and mecs_5.grouping, respectively, (these files allow us to sample the instrument resolution with the same number of channels, 5, at all energies). Given the large number of counts (allowing to reach in each rebinned channel an uncertainty around $2 \%$, i.e. the accuracy level of the MECS calibration for bright source) we consider only the MECS2 unit. HPGSPC data were extracted using SAXDAS (hpproducts V3.0.0). PDS data were extracted using XAS. The HPGSPC and PDS background was estimated with standard procedures using off-axis blank fields. HPGSPC and PDS data were grouped in bins which are (nearly) logarithmically spaced. Given the large number of counts we carried out the spectral analysis in the energy ranges where the instrumental responses are best known: $0.3-4 \mathrm{keV}$ for the LECS, 1.8-10 keV for the MECS, 8-20 keV for the HPGSPC and 15-200 keV for the PDS. We used the publicly available 
Table 1. Summary of BeppoSAX observations.

\begin{tabular}{|c|c|c|c|c|c|c|c|c|c|}
\hline $\begin{array}{l}\text { Obs. } \\
\text { num. }\end{array}$ & $\begin{array}{l}\text { Start } \\
\text { time } \\
\text { MJD }\end{array}$ & $\begin{array}{c}\text { LECS } \\
\text { Exp. time } \\
\text { (s) }\end{array}$ & $\begin{array}{c}\text { LECS } \\
\text { Count rate } \\
\mathrm{c} \mathrm{s}^{-1}\end{array}$ & $\begin{array}{c}\text { MECS } \\
\text { Exp. time } \\
\text { (s) }\end{array}$ & $\begin{array}{c}\text { MECS } \\
\text { Count rate } \\
\mathrm{c} \mathrm{s}^{-1}\end{array}$ & $\begin{array}{l}\text { HPGSPC } \\
\text { Exp. time } \\
\text { (s) }\end{array}$ & $\begin{array}{c}\text { HPGSPC } \\
\text { Count rate } \\
\mathrm{c} \mathrm{s}^{-1}\end{array}$ & $\begin{array}{c}\text { PDS } \\
\text { Exp. time } \\
\text { (s) }\end{array}$ & $\begin{array}{c}\text { PDS } \\
\text { Count rate } \\
\mathrm{c} \mathrm{s}^{-1}\end{array}$ \\
\hline 1 & 50961.554 & 11540 & 24.4 & 23641 & 19.6 & 11544 & 11.2 & 11973 & 2.7 \\
\hline 2 & 50974.535 & 4151 & 20.1 & 7916 & 17.5 & 2769 & 1.4 & 2396 & 1.4 \\
\hline 3 & 50982.365 & 16495 & 22.9 & 27339 & 20.0 & 11160 & 0.6 & 10281 & 0.2 \\
\hline 4 & 50987.250 & 19388 & 26.7 & 31500 & 22.2 & - & - & 13282 & $<0.2^{*}$ \\
\hline 5 & 51002.854 & 15214 & 19.7 & 27142 & 17.0 & - & - & 12037 & 0.3 \\
\hline
\end{tabular}

Count rates are in the energy bands used in the spectral analysis (see text).

* $3 \sigma$ upper limit.

calibration files of January 2000 and XSPEC 11.1.0. We added a systematic error of $2 \%$ to account for systematic uncertainties in the calibrations. This is in line with previous studies of bright galactic sources (e.g. Oosterbroek et al. 2001). A variable normalisation factor was used in the spectral analysis to account for the mismatch in the (small) absolute flux calibration between the different BeppoSAX instruments (Fiore et al. 1999).

As can be observed from Table 1, the PDS and HPGSPC count rates changed considerably across the observational campaign with a drop by a factor of larger than 10. At lower energies the count rates underwent significant, though considerably smaller, variations. A simple and model-independent way of looking at these variations is to construct a color-color diagram. We considered three energy bands: Soft (S, 2-6 keV, MECS), Medium (M, 6-10 keV, MECS) and Hard (H, 15-50 keV, PDS). We defined a soft color obtained as the ratio $\mathrm{M} / \mathrm{S}$ and a hard color as H/M. The color-color diagram for the entire set of observations is shown in Fig. 2. The first observation (filled dots) is clearly separated from the others both in terms of soft and hard colors; also the second observation (open dots) is harder than the others. The remaining three observations lay all in the same region with low soft color.

We first tried single component models to fit the data, but all of them provided poor fits (reduced $\chi^{2} \gtrsim 20$ ). We tried different two-component models made by a disk black body component plus a power law, a cut-off power law or a Comptonisation tail. We first focus on the first model and discuss in the following the other two.

As reported by White et al. (1998) using ASCA data and by Vasiliev et al. (2000) using RXTE data, the standard model for black hole candidates consisting of a disk black body (Mitsuda et al. 1984) plus a power law and a Gaussian iron line provides an adequate fit to those data. As reported in Table 2 (see also Fig. 3), this model provided also an adequate fit to the entire BeppoSAX dataset. Absorbed and unabsorbed fluxes in different energy bands are reported in Table 4.

This model parametrisation allows us to monitor the spectral evolution of XTE 2012+381 (see Fig. 4) and to compare the results with observations from other instruments. For the column density we limit our analysis to the BeppoSAX data and model it with the new

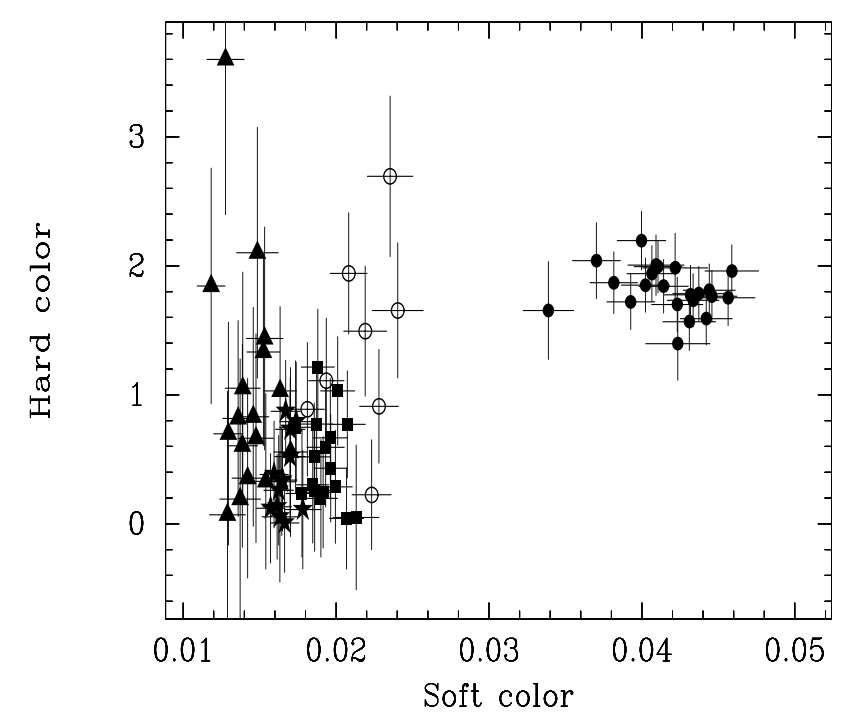

Fig. 2. Color-color digram for the five BeppoSAX observations of XTE J2012+381. Filled dots, empty dots, filled squares, filled stars and filled triangles mark the colors of the 1st, 2nd, $3 \mathrm{rd}, 4$ th and 5 th observations, respectively. Soft color data are obtained as the ratio of $6-10 \mathrm{keV}$ to $2-6 \mathrm{keV}$ (background subtracted) counts in the MECS; hard color data as the ratio of $15-50 \mathrm{keV}$ to $6-10 \mathrm{keV}$ (background subtracted) counts in the PDS and MECS, respectively. Counts are evaluated in a 1000 s time bin.

XSPEC model TBABS (which includes updates to the photoionisation cross section, revised abundances of the interstellar medium, and presence of interstellar grains and the H2 molecule; Wilms et al. 2000). This new model gives significantly better fits then the usual WABS model and a slightly lower column density value. Across the BeppoSAX observational campaign the value of the column density remains unchanged with a mean value of $(1.27 \pm 0.02) \times 10^{22} \mathrm{~cm}^{-2}$. This is consistent with the Galactic value of $1.2 \times 10^{22} \mathrm{~cm}^{-2}$ (Dickey \& Lockman 1990). In the analysis of the other spectral parameters we include also the values derived from RXTE observations (Fig. 5). The parameters of the soft spectral component display small but significant variations: the temperature follows closely the flux evolution whereas the radius shows a steep increase from the first observation and then settles to a constant value of $R=39.5 \pm 0.3 \mathrm{~km}$ (see Fig. 5). The iron line properties remain constant with a mean centroid $E=5.9 \pm 0.3 \mathrm{keV}$ and a mean line 
Table 2. Spectral parameters (disk black body + power law + Gaussian line) the BeppoSAX observations.

\begin{tabular}{lcccccccc}
\hline Obs. & $\begin{array}{c}N_{\mathrm{H}} \\
\left(10^{22} \mathrm{~cm}^{-2}\right)\end{array}$ & $\begin{array}{c}k T \\
(\mathrm{keV})\end{array}$ & $\begin{array}{c}\text { Disk radius } \\
(\mathrm{km})\end{array}$ & $\begin{array}{c}\text { Power law } \\
\text { photon index }\end{array}$ & $\begin{array}{c}\text { Line energy } \\
(\mathrm{keV})\end{array}$ & $\begin{array}{c}\text { Line width } \\
(\mathrm{keV})\end{array}$ & $\begin{array}{c}\text { Line } E W \\
(\mathrm{eV})\end{array}$ & $\begin{array}{c}\chi^{2} \\
\text { red. }(\mathrm{d} . \mathrm{o} . \mathrm{f} .)\end{array}$ \\
\hline 1 & $1.30 \pm 0.02$ & $0.75 \pm 0.01$ & $33.3 \pm 0.7$ & $2.22 \pm 0.05$ & $5.95 \pm 0.15$ & $1.13 \pm 0.15$ & 343 & $1.09(244)$ \\
2 & $1.24 \pm 0.02$ & $0.72 \pm 0.01$ & $38.2 \pm 1.3$ & $2.12 \pm 0.28$ & $5.92 \pm 1.50$ & $1.26 \pm 0.71$ & 409 & $1.16(172)$ \\
3 & $1.32 \pm 0.06$ & $0.73 \pm 0.01$ & $38.5 \pm 0.9$ & $3.07 \pm 0.29$ & $5.89 \pm 1.90$ & $0.88 \pm 1.18$ & 145 & $0.95(197)$ \\
$4^{\ddagger}$ & $1.27 \pm 0.03$ & $0.76 \pm 0.01$ & $37.9 \pm 0.7$ & $3.33 \pm 1.40$ & $6.00($ fixed) & $1.60 \pm 0.35$ & 297 & $1.15(152)$ \\
5 & $1.25 \pm 0.02$ & $0.72 \pm 0.01$ & $38.9 \pm 1.1$ & $1.82 \pm 0.46$ & $5.84 \pm 0.88$ & $1.10 \pm 0.73$ & 320 & $0.98(150)$ \\
\hline
\end{tabular}

All the errors are at $90 \%$ confidence level for one interesting parameter.

\# We assumed a distance of $10 \mathrm{kpc}$ and an inclination of 0 degrees.

¥ The spectral modelling of this observation turned to be extremely problematic. Only by fixing the value of the iron line (to $6.0 \mathrm{keV}$ ) a reasonably good fit can be achieved.

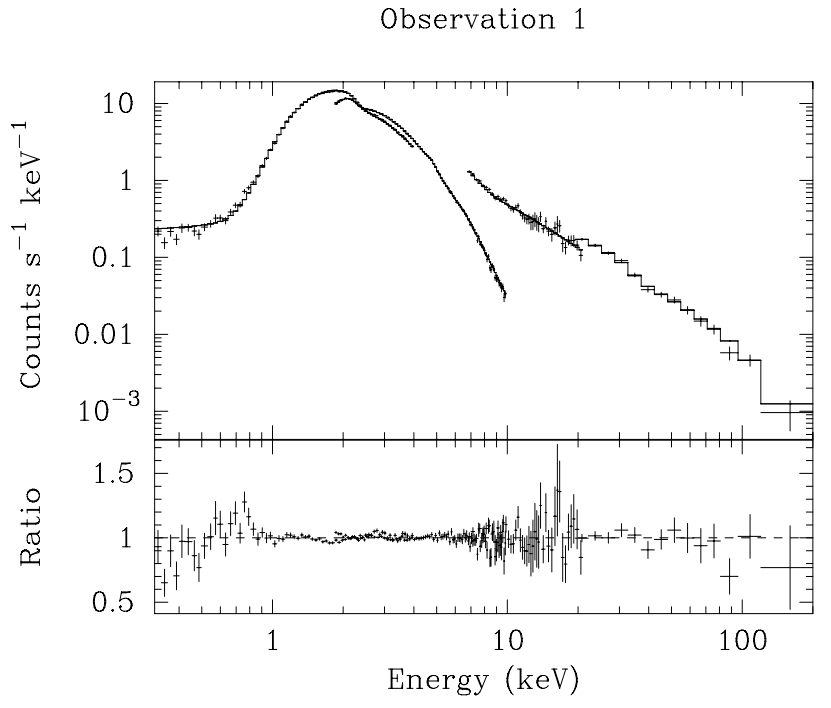

Fig. 3. X-ray spectrum of the first BeppoSAX observation of XTE J2012+381 with the disk black body power law model. Residuals from the fit are shown in the lower panel.

width $(\sigma) W=1.2 \pm 0.2 \mathrm{keV}$ (these values are based only on BeppoSAX analysis since Vasiliev et al. keep the iron line energy fixed at $6.4 \mathrm{keV}$ ). Broad and redshifted iron lines are rare among $\mathrm{X}$-ray binaries. This is potentially extremely interesting however we note that the power law and the disk black body components become comparable just around $6 \mathrm{keV}$. We speculate that part of the line width is to ascribe to a non-perfect matching of the two components. The same problem is also present in the RXTE data.

The slope of the power law component remained constant in the first part of the outburst up to MJD 50981 (with $\Gamma=2.2 \pm 0.1$ ), steepened in the second part of the outburst ( $\Gamma=3.2 \pm 0.3$; see Fig. 5$)$ and it hardened again around MJD 51000. This change occurs simultaneously with the reflare in the outburst light curve. As a result of this, the extrapolated flux at high energies $(>20 \mathrm{keV})$ drops smoothly by a factor of $\gtrsim 800$ (see Table 4 ). This value is based on the hard flux derived from spectral fits (and therefore for the third and fourth observations is extrapolated). If we consider the count rate variation the drop is by a factor of $>10$.
In the last BeppoSAX observation a re-hardening is observed (see Figs. 4, 5). In this observation the power law is as hard as in the first two observations and also RXTE observations contiguous (in time) provide similar hard power laws $(\Gamma=1.9 \pm 0.4$; see Fig. 5$)$. It is interesting to note that the flux level at which this re-hardening occurs is just below a threshold of $\sim 9 \mathrm{ASM} \mathrm{c} \mathrm{s}{ }^{-1}$ (see Fig. 4), which is also the threshold at which the the first flare ends and the reflare starts.

We also tried different models such as PEXRAV (consisting of an exponentially cut-off power law plus the reflected component from neutral material; Magdziarz \& Zdziarski 1995), or Comptonisation models (e.g. COMPTT, Titarchuk 1994), together with a disk black body and an iron line. The PEXRAV model provides an equally good fit to the first observation $\left(\chi_{\text {red }}^{2}=1.09\right)$ but XSPEC fails to converge for the second one. Moreover, the reflected fraction is very low. For these reasons we do not consider this model. The COMPTT model gives $\chi_{\text {red }}^{2}=1.09,1.12,0.92,1.03,0.98$ for the five observations, respectively. The COMPTT model provides a better fit with respect to the power law model on the entire dataset at $99.4 \%$ confidence level (estimated by means of an F-test). We decide to discuss first the disk black body plus power law model in order to include also the RXTE data analysed by Vasiliev et al. (2000). In Table 3 we report the parameters of the COMPTT model only, with the column density, soft component and iron line remaining almost the same. A clear trend can also be observed in this spectral decomposition following closely the source flux and with the hardest observations having the smallest input soft photon (Wien) temperature $\left(T_{0}\right)$ and the highest plasma temperature $T_{\mathrm{C}}$.

We also considered a model with a cut-off power law. In this case we have problems in disentangling the power law photon index and the cut-off energy. What occurred is that the power law becomes steeper and steeper and the cut-off energy lower and lower (e.g. for the second observation we obtained a power law photon index -0.5 and a cut-off energy of $10 \mathrm{keV}$ ). In order to monitor the cut-off energy in the different spectral states we decided to fix the power law index to 2 and to derive the cut-off energy. This energy turned out to decrease (as expected) with the softening of the spectrum (see Table 3). 
Table 3. Parameters of the Comptonisation and cut-off power law models.

\begin{tabular}{cccc|c}
\hline Obs. & $\begin{array}{c}T_{0} \\
(\mathrm{eV})\end{array}$ & $\begin{array}{c}T_{\mathrm{C}} \\
(\mathrm{keV})\end{array}$ & $\tau$ & $\begin{array}{c}E_{\text {cutoff }} \\
(\mathrm{keV})\end{array}$ \\
\hline 1 & 8 & 152.5 & 0.09 & 205 \\
2 & 62 & 96.6 & 0.22 (fixed) & 134 \\
3 & 26 & 45.4 & 0.17 & 8.8 \\
4 & 46 & 14.0 & 0.08 & 8.0 \\
5 & 31 & 169.5 & 0.22 & $>80$ \\
\hline
\end{tabular}

Fitting the second observation proved problematic and we have to fixed the value of at least one parameter to make the fit converge. Given the similarities between the second and the fifth observation, we decided to fix the optical depth $\tau$ in the COMPTT model to 0.22 . The fit in any case was highly unstable and we cation the reader on the results obtained.

\subsection{Timing analysis}

For each observation, we produce a rms normalised power spectrum of the 1.8-10 keV light curve to study the timing properties of the system. Both MECS units are used in this case. Power spectra do not show prominent noise components. No periodicities or quasi-periodicities are present with an upper limit of $\$ 5 \%$ over the $10^{-2}-1 \mathrm{~Hz}$ range for the five observations discussed; this limit increases to $\sim 60 \%$ at $10^{-4} \mathrm{~Hz}$. In order to better characterise the spectral states of XTE J2012+381 we compute the fractional rms variability (for frequencies higher than $1 \mathrm{~Hz}$ ): this is $<4.7 \%$ ( $3 \sigma$ level) $,<5.4 \%,<6.4 \%,<8.6 \%$ and $<7.6 \%$ for the five MECS observations, respectively. A more detailed analysis is carried out with the RXTE light curves. By averaging observations between MJD 50962 and MJD 50987, Vasiliev et al. (2000) obtained a power spectrum dominated by the very low frequency noise with a $\nu^{-1}$ dependence. The fractional $\mathrm{rms}$ variability was at a level of $\sim 2 \%$. An indication of the increase of the fractional rms with energy was also found. No quasi-periodic oscillations were detected. Here we reanalysed the same set of data splitting the RXTE data set into three periods. For the first period (between MJD 50962 and MJD 50981 encompassing the first two BeppoSAX observations) we derive a rms variability of $1.7 \%$. For the 4 RXTE observations between MJD 50986 and MJD 51001 (including the third and fourth BeppoSAX observation) the fractional rms variability raised to $3 \%$. Finally the four last RXTE observations in between MJD 51007 and MJD 51024 provided only an upper limit of $<1.2 \%$.

\subsection{Quiescent emission}

The field containing XTE J2012+381 was monitored a few times by ROSAT when observing the reflection nebula NGC 6888. Three observations were carried out with the HRI detector for a total observing time of $109 \mathrm{ks}$. One observation was carried out with the PSPC detector for $8 \mathrm{ks}$. In none of the observations XTE J2012+381 was detected. The tightest upper limit is provided by the three
HRI observations summed together. Assuming the same column density observed during the 1998 outburst, the extrapolated $3 \sigma$ upper limit on the quiescent $0.1-2.4 \mathrm{keV}$ (unabsorbed) luminosity is $\sim 2 \times 10^{33} \mathrm{erg} \mathrm{s}^{-1}$ for a Crab spectrum and a distance of $10 \mathrm{kpc}^{1}$. This limit is fairly high and it is mainly due to the low energy pass band together with the high column density. This limit alone cannot allow us to disentangle the nature of the compact object in XTE J2012+381 (e.g. Campana \& Stella 2000).

\section{Discussion}

BeppoSAX observations as well as other X-ray observations made possible the identification of an ultra-soft component $(k T \sim 0.7 \mathrm{keV})$ together with a hard energy tail (photon index $\sim 2$; see also White et al. 1998; Vasiliev et al. 2000) extending up to $200 \mathrm{keV}$ in the outburst X-ray spectrum of XTE J2012+381. Spectral variability was observed with the hard power law count rate decreasing by a factor $\gtrsim 10$ in the $20-200 \mathrm{keV}$ band and a factor of $\gtrsim 800$ in flux according to the fitted spectra. These characteristics, together with the lack of X-ray bursts and coherent pulsations, clearly suggest a black hole nature of XTE J2012+381.

The absorbed $0.1-200 \mathrm{keV}$ luminosity during the BeppoSAX observations was at a level of $\sim 4 \times$ $10^{37} d_{10}^{2} \operatorname{erg~s}^{-1}$ and showed only modest variations $(\sim 30 \%)$ across the different observations. Given the large column density, removing the effects of the interstellar absorption results in a substantially higher luminosity: the $0.1-200 \mathrm{keV}$ unabsorbed luminosity is $1.3,1.1,2.1,1.6$, $1.0 \times 10^{38} d_{10}^{2} \mathrm{erg} \mathrm{s}^{-1}$, however.

\subsection{Taxonomy of source states}

Different spectral states have been identified in black hole candidates (BHCs) as their spectral and timing properties vary along with their X-ray luminosity (for a review see e.g. van der Klis 1995). In the low state (LS) BHCs are characterised by a power law spectrum $(\Gamma \sim 1.5-2)$ and (sometimes) a weak disk component $(k T \lesssim 1 \mathrm{keV})$. Strong rapid aperiodic variability with fractional $\mathrm{rms}$ amplitude of $20-50 \%$ and a break frequency below $1 \mathrm{~Hz}$ is also observed. At increasing X-ray fluxes, the source enters the intermediate state (IS), characterised by a steep power law $(\Gamma \sim 2-3)$ plus a disk component. The timing properties present a broad band noise with $\sim 5-20 \%$ rms and a break frequency higher than 1-10 Hz. The high state (HS) is dominated by the disk component with a weak and steep power law $(\Gamma \sim 2-3)$. A few percent rms characterise the temporal variability and this is its main difference with the IS. Finally, a very high state (VHS) has been observed

\footnotetext{
${ }^{1}$ An upper limit on the distance $d \lesssim 12 \mathrm{kpc}$ comes from the requirement that XTE J2012+381 lies within the Galaxy. The column density value inferred from the BeppoSAX data, being compatible with the full galactic value, favours a distant object. In the following we assume a value of $d=d_{10} 10 \mathrm{kpc}$.
} 


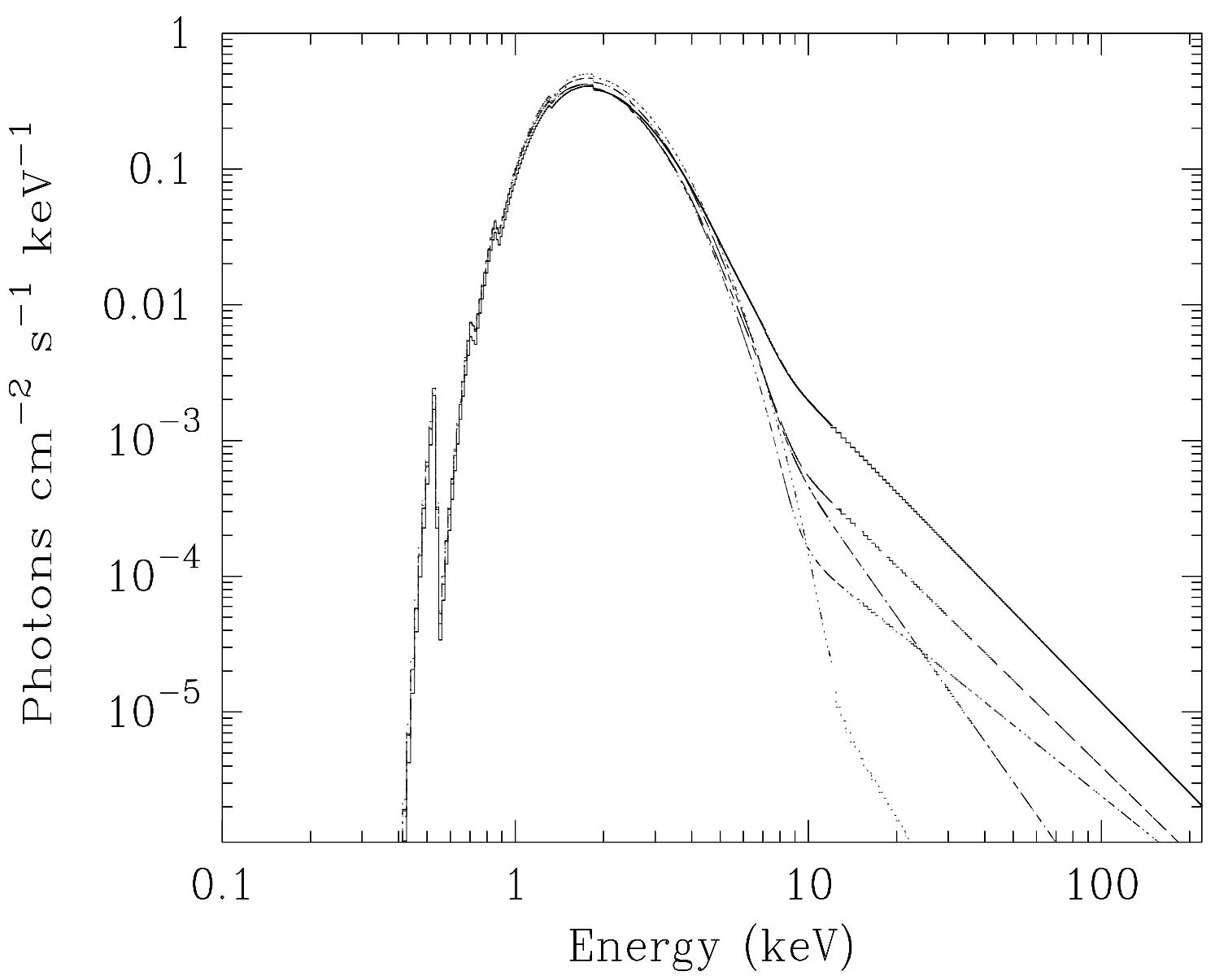

Fig. 4. Absorbed model spectra of the five BeppoSAX observations of XTE J2012+381, assuming a disk black body plus power law model. Spectra from different observations are indicated by different line styles (1st: continuous; 2nd: dashed; 3rd: dot-dotdot-dashed; 4th: dotted; 5th: dot-dashed style). Note that for the fifth observation the extrapolation at high energies is rather uncertain.

Table 4. X-ray (absorbed) fluxes for the BeppoSAX observations with power law fit.

\begin{tabular}{ccccccc|c}
\hline Obs. & $\begin{array}{c}0.1-1 \mathrm{keV} \\
\left(10^{-11} \mathrm{cgs}\right)\end{array}$ & $\begin{array}{c}2-10 \mathrm{keV} \\
\left(10^{-9} \mathrm{cgs}\right)\end{array}$ & $\begin{array}{c}20-200 \mathrm{keV} \\
\left(10^{-11} \mathrm{cgs}\right)\end{array}$ & $\begin{array}{c}0.1-200 \mathrm{keV} \\
\left(10^{-9} \mathrm{cgs}\right)\end{array}$ & $\begin{array}{c}\text { DBB flux } 2-10 \mathrm{keV} \\
\left(10^{-9} \mathrm{cgs}\right)\end{array}$ & $\begin{array}{c}\text { PL flux 2-10 keV } \\
\left(10^{-9} \mathrm{cgs}\right)\end{array}$ & $\begin{array}{c}0.1-200 \mathrm{keV} \text { UNABS } \\
\left(10^{-8} \mathrm{cgs}\right)\end{array}$ \\
\hline 1 & 1.39 & 2.47 & 48.25 & 3.96 & 1.92 & 0.52 & 1.08 \\
2 & 1.63 & 2.10 & 15.44 & 3.16 & 1.96 & 0.14 & 0.90 \\
3 & 1.66 & 2.39 & 2.80 & 3.38 & 2.17 & 0.22 & 1.77 \\
4 & 1.80 & 2.66 & 0.24 & 3.67 & 2.60 & 0.06 & 1.30 \\
5 & 1.62 & 2.00 & 8.00 & 2.94 & 1.96 & 0.04 & 0.86 \\
\hline
\end{tabular}

Fluxes are derived from spectral fits. In particular, the $20-200 \mathrm{keV}$ flux of the fourth observation is extrapolated from the model, whereas the PDS provided only an upper limit.

in a few BHCs, with a spectrum similar to the one in the intermediate state (disk component with $k T \sim 1-2 \mathrm{keV}$ and power law with $\Gamma \sim 2.5$ ), and with band limited noise at $1-15 \%$ rms level. Quasi-periodic oscillations at $1-10 \mathrm{~Hz}$ are often seen (e.g. Miyamoto et al. 1991; Homan et al. 2001). This classification scheme is one-dimensional with transitions following LS $\rightarrow$ IS $\rightarrow$ HS $\rightarrow$ VHS for increasing mass accretion rates. Homan et al. (2001) analysed the RXTE data of the BHC XTE J1550-564 and noted that the simple correlation of states with accretion rate breaks down. Given the similarity of the IS and the VHS, they concluded that the VHS is an instance of the IS. Based on this, the classification scheme that they propose envisages a two-dimensional behaviour with the count rate and the spectral hardness as phenomenological parameters. In this framework the difference between the IS and the VHS is reduced to a difference in the count rates. (The two physical parameters are suggested to be the mass accretion rate and extent of a Comptonising corona, responsible for the hard component.)

In all our observations the fractional rms variability is low and the disk (soft) component is strong comprising the great majority of the source luminosity. These properties likely exclude that XTE $2012+381$ entered the LS. In the first two observations of XTE J2012+381 a hard power law with photon index $\sim 2$ (up to $\sim 200 \mathrm{keV}$ ) is clearly detected 


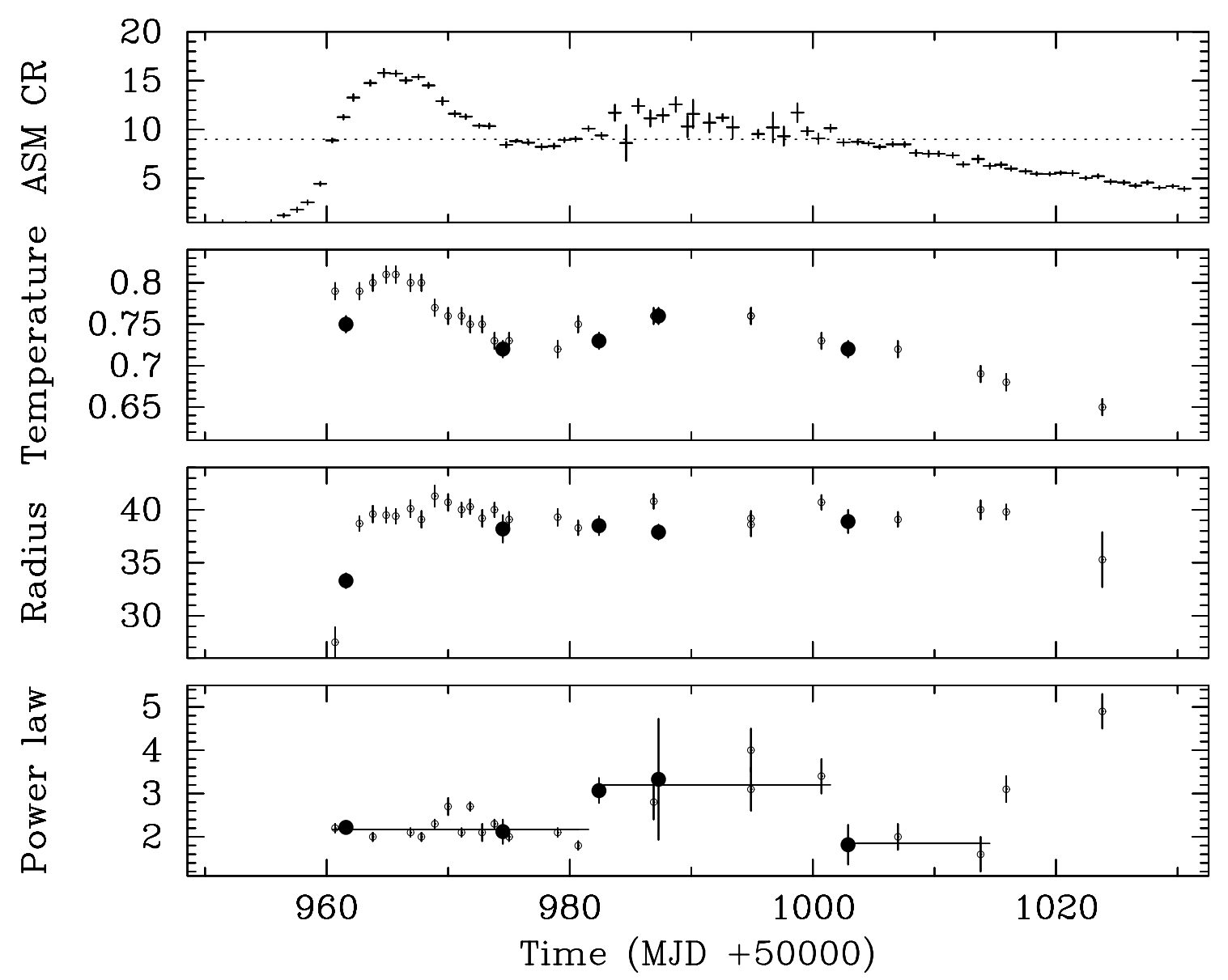

Fig. 5. Evolution of the light curve and spectral parameter. From top to bottom, count rate, temperature and radius of the disk black body component, and power law photon index. In the first panel a rough indication of the flux at which spectral changes occur is drawn with a dotted line. In the lowest panel a fit with a constant is overlaid for the three periods discussed in the text. Filled dots indicate values derived from BeppoSAX observations. Small open dots indicate values obtained with RXTE (Vasiliev et al. 2000).

(see Fig. 4). These characteristics together with the timing properties outlined above likely place XTE $2012+381$ on a hard branch (i.e. the IS/VHS). In the third and fourth observations XTE J2012+381 has a much softer spectrum (with a power law photon index $\sim 4$ barely detected in the PDS up to $\sim 50 \mathrm{keV}$ in the third) indicating that the source entered the HS. This is further corroborated by the fact that in the fourth observation the count rate is the highest. Finally in the last observation the source came back with a relatively hard power law (even if affected by a large uncertainty) and a small rms variability, likely indicating that it returned to the hard branch. The source count rate being much larger in the first two observations than in the last, in the "old" classification the first two might be ascribed to the VHS and the last to the IS.

\subsection{Comparison with other black hole candidates outbursts}

Finally, we note that the XTE J2012+381 outburst evolution shares similar characteristics with well known BHC transients, even though the characteristic timescales are shorter: $i$ ) after the outburst peak (around May 22th 1998, MJD 50955.6) the RXTE-ASM light curve decayed exponentially with an $e$-folding time of $16 \mathrm{~d}$. This time is 24,31 and $30 \mathrm{~d}$ for A 0620-00, GS 1124-684, GS $2000+251$, respectively. $i i)$ a secondary peak occurred $\sim 30$ later (Jun. 26th 1998, MJD 50990.6), reaching a level of $\sim 75 \%$ the outburst peak. A secondary peak occurred 55 , 60-75 and 70 d after maximum in A 0620-00, GS 1124684, GS 2000+251, respectively (e.g. Chen et al. 1993). iii) after the second peak the $\mathrm{X}$-ray light curve decayed exponentially with an $e$-folding time of $35 \mathrm{~d}$, i.e. more slowly than after the first peak. An $e$-folding time of 20 , 37 and $30 \mathrm{~d}$ was observed in A 0620-00, GS 1124-684 and GS 2000+251, respectively. iv) there was evidence for a possibly third broad peak about $150 \mathrm{~d}$ after the outburst peak (Oct. 24th 1998, MJD 51110.6). The same feature was detected in A 0620-00 ( 200 d after the outburst) and in GS $2000+251(\sim 70 \mathrm{~d})$.

Concerning the spectral evolution, the low energy portion of the spectra remained basically unchanged during the outburst evolution. At high energies, the power law tail is hard and steepens later on. This spectral behaviour 
is similar to the one observed in GS 1124-68 (Nova Muscae 1991; Tanaka 1992), though (again) on a shorter temporal base: around the outburst maximum the spectrum was hard but it was softer at the time of the secondary outburst. In GS 1124-68 a hard tail was observed again in the observations following the secondary maximum, about $120 \mathrm{~d}$ after the main peak, similarly to what occurred in XTE $2012+381 \sim 40 \mathrm{~d}$ after the main peak. These similarities further strenghten the identification of XTE 2012+381 with a black hole candidate.

\section{Conclusions}

BeppoSAX observations that covered the 1998 outburst of XTE J2012+381 found several similarities with known BHCs. In particular, the X-ray spectrum could be well described by an ultrasoft component (disk black body) with the addition of a variable hard power law.

One can derive an upper limit on the black hole mass by interpreting the inner disk radius from the spectral fits as the last marginally stable orbit. The lowest values are obtained for the first observation with an equivalent radius $R=33 \mathrm{~km}$. For a disk inclination of $0^{\circ}$, we derive $M \gtrsim 3.7 d_{10} M_{\odot}\left(M \gtrsim 22 d_{10} M_{\odot}\right)$ for a non-rotating (maximally rotating) black hole. Notice that the absolute value inferred for the disk radius, due to the approximations in the disk black body model used, is to be considered also a lower limit (Merloni et al. 2000).

One of the main results of the present paper is the different spectral states observed during the outburst evolution of XTE $2012+281$. The column density, the parameters of the soft component and iron line remained almost constant. On the other hand the hard tail changed considerably across different observations. During the first flare (between MJD 50960 and 50982) the X-ray spectrum was hard with a power law index $\Gamma=2.2 \pm 0.1$. In the second flare (between MJD 50982 and 51002) the $\mathrm{X}$-ray spectrum firstly became soft and then it rehardened (between MJD 51003 and 51014). At the outburst end, there is a hint for a further softening. In passing, we note that BeppoSAX and RXTE spectral fits are in good agreement, despite the poorer resolution of RXTE spectra. Moreover, our timing analysis put in evidence a higher rms variability in the interval during which the spectrum of XTE $2012+281$ is soft.

The transition from a hard to a soft and then back to a hard state occurs around the same count rate in the RXTE-ASM around $\left(\sim 9 \mathrm{c} \mathrm{s}^{-1}\right)$. Clearly for such an absorbed source the ASM cannot provide a fair estimate of the source luminosity. Comparing BeppoSAX (unabsorbed) luminosities derived from the spectral decomposition we see that this threshold is around $10^{-8} \mathrm{erg} \mathrm{s}^{-1} \mathrm{~cm}^{-2}$, i.e. $10^{38} d_{10 \mathrm{kpc}}^{2} \mathrm{erg} \mathrm{s}^{-1}$. Above this flux level, during the first flare the spectrum of XTE $2012+281$ is hard, whereas during the second flare above the same flux level the source spectrum is soft. Simultaneously, the rms fractional variability increases only slightly from $\sim 2 \%$ to $\sim 3 \%$.
This likely indicate that state transitions in XTE $2012+281$ are not driven only by mass accretion rate and at least one other parameter is needed. In particular, the soft component remains almost stable during the BeppoSAX and RXTE observational campaigns (within a factor of a few) whereas the hard component varied considerably. In this regard we note that spectral parameters such as the plasma temperature $T_{\mathrm{C}}$ or the high energy cut-off vary considerably across the transition strengthening the idea that the second parameter involved in state transitions is related to a hot corona.

Acknowledgements. This research has made use of SAXDAS linearised and cleaned event files (Rev.2.0) produced at the BeppoSAX Science Data Center. This work was partially supported through ASI grants. J. Homan is acknowledged for useful comments. TB thanks the Cariplo Foundation for support.

\section{References}

Boella, G., Butler, R. C., Perola, G. C., et al. 1997a, A\&AS, 122, 299

Boella, G., Chiapetti, L., Conti, G., et al. 1997b, A\&AS, 122, 327

Campana, S., \& Stella, L. 2000, ApJ, 541, 849

Chen, W., Shrader, C. R., \& Livio, M. 1997, ApJ, 491, 312

Dickey, J. M., \& Lockman, F. J. 1990, ARA\&A, 28, 215

Fiore, F., Guainazzi, M., \& Grandi, P. 1999, Cookbook for the BeppoSAX NFI spectral analysis, available at http://www.sdc.asi.it/software/index.html

Frontera, F., Costa, E., dal Fiume, D., et al. 1997, A\&AS, 122, 357

Hjellming, R. M., Rupen, M. P., \& Mioduszewski, A. J. 1998a, IAUC, 6924

Hjellming, R. M., Rupen, M. P., \& Mioduszewski, A. J. 1998b, IAUC, 6932

Homan, J., Wijnands, R., van der Klis, M., et al. 2001, ApJS, 132,377

Hynes, R. I., \& Roche, P. 1998, IAUC, 6932

Hynes, R. I., Roche, P., Charles, P. A., \& Coe, M. J. 1999, MNRAS, 305, L49

Magdziarz, P., \& Zdziarski, A. A. 1995, MNRAS, 273, 837

Manzo, G., Giaresso, S., Santangelo, A., et al. 1997, A\&AS, 122,341

Marshall, F., \& Strohmayer, T. 1998, IAUC, 6922

Merloni, A., Fabian, A. C., \& Ross, R. R. 2000, MNRAS, 313, 193

Mitsuda, K., Inoue, H., Koyama, K., et al. 1984, PASJ, 36, 741

Miyamoto, S., Kimura, K., Kitamoto, S., Dotani, T., \& Ebisawa, K. 1991, ApJ, 383, 784

Oosterbroek, T., Barret, D., Guainazzi, M., \& Ford, E. C. 2001, A\&A, 366, 138

Parmar, A. N., Martin, D. D. E., Bavdaz, M., et al. 1997, A\&AS, 122, 309

Remillard, R., Levine, A., \& Wood, A. 1998, IAUC, 6920

Tanaka, Y., \& Shibazaki, N. 1996, ARA\&A, 34, 607

Titarchuk, L. 1994, ApJ, 434, 570

van der Klis, M. 1995, The Lives of the Neutron Stars, NATO Proc., ed. M. A. Alpar, U. Kiziloglu, \& J. van Paradijs (Kluwer, Dordrecht), 301

Vasiliev, L., Trudolyubov, S., \& Revnivtsev, M. 2000, A\&A, 362, L53

White, N. E., Ueda, Y., Dotani, T., \& Nagase, F. 1998, IAUC, 6927

Wilms, J., Allen, A., \& McCray, R. 2000, ApJ, 542, 914 